\title{
Upregulation of HIF-1 $\alpha$ protein induces mitochondrial autophagy in primary cortical cell cultures through the inhibition of the mTOR pathway
}

\author{
GU GONG $^{1 *}$, LING HU $^{1 *}$, YINGHAI LIU ${ }^{1}$, SHURONG BAI ${ }^{1}$, XUEMEI DAI ${ }^{1}$, \\ LIANG YIN ${ }^{1}$, YANGYANG SUN ${ }^{1}$, XIAOWU WANG ${ }^{2}$ and LICHAO HOU ${ }^{3}$ \\ ${ }^{1}$ Department of Anesthesiology, General Hospital of the People's Liberation Army Chengdu Military Region, \\ Chengdu, Sichuan $610083 ;{ }^{2}$ Center of Cardiovascular Surgery, Guangzhou General Hospital of \\ Guangzhou Military Command, Guangzhou, Guangdong 510010; ${ }^{3}$ Department of Anesthesiology, \\ Xijing Hospital, Fourth Military Medical University, Xi'an, Shaanxi 710032, P.R. China
}

Received December 30, 2013; Accepted June 26, 2014

DOI: $10.3892 /$ ijmm.2014.1850

\begin{abstract}
Cerebral ischemia/reperfusion (I/R) can induce neuronal death, particularly in the hippocampal formation (HF). Molecular genetic studies have suggested that the activities of the transcription factor, hypoxia-inducible factor-1 $\alpha$ (HIF-1 $\alpha$ ), are closely linked to ischemia-induced neuronal death. However, the mechanisms through which HIF-1 $\alpha$ functions remain poorly understood. In this study, primary cortical neurons were subjected to oxygen-glucose deprivation (OGD) to establish a cell model of OGD/reperfusion (RP). HIF-1 $\alpha$ mRNA and protein expression was measured by qRT-PCR and western blot analysis. Cell proliferation was detected by MTT assay. Flow cytometric analysis was used to detect cell apoptosis and changes in mitochondrial mass. The expression of LC3-I and LC3-II was examined by western blot analysis. We found that HIF-1 $\alpha$ increased cell proliferation and decreased cell apoptosis in our cell model of OGD/RP using cultured neonatal rat cortical neurons. The overexpression of
\end{abstract}

Correspondence to: Dr Xiaowu Wang, Center of Cardiovascular Surgery, Guangzhou General Hospital of Guangzhou Military Command, 111 Liuhua Road, Guangzhou, Guangdong 510010, P.R. China

E-mail: xiaowu_wanggz@163.com

Dr Lichao Hou, Department of Anesthesiology, Xijing Hospital, Fourth Military Medical University, 15 Changle West Road, Xi'an, Shaanxi 710032, P.R. China

E-mail: lichao_hou@163.com

${ }^{*}$ Contributed equally

Abbreviations: I/R,cerebral ischemia/reperfusion; HIF-1 $\alpha$, hypoxiainducible factor-1 $\alpha$; OGD, oxygen-glucose-deprivation; HF, hippocampal formation; siRNA, small interference RNA

Key words: cortical cell culture, oxygen-glucose deprivation, hypoxia-inducible factor- $1 \alpha$, mitochondrial autophagy
HIF-1 $\alpha$ significantly induced changes in mitochondrial mass and mitochondrial autophagy in cortical neurons. Moreover, the inhibition of HIF-1 $\alpha$ markedly suppressed cell proliferation and mitochondrial autophagy. We also demonstrated that the HIF-1 $\alpha$-induced mitochondrial autophagy was accompanied by the inhibition of the mTOR pathway. This study provides direct in vitro evidence that HIF-1 $\alpha$ overexpression triggers mitochondrial autophagy, thereby increasing neuronal survival. Our results highlight a novel target molecule toward which anti-ischemic neuroprotective effects can be applied.

\section{Introduction}

Ischemic brain injury is an important disorder that threatens human health and life (1). Ischemic brain injury can cause high mortality and is the third leading cause of mortality among Americans (2). In addition to high mortality, ischemic brain injury can also lead to long-term disability (3). Cerebral ischemia/reperfusion (I/R) can induce neuronal death, particularly in the hippocampal formation (HF) (4). Molecular genetic studies have suggested that the activities of the transcription factor, hypoxia-inducible factor-1 $\alpha$ (HIF-1 $\alpha$ ), are closely linked to ischemia-induced neuronal death $(5,6)$. Cerebral ischemia results in low oxygen delivery and a decrease in the adenosine triphosphate (ATP) concentration (7), and the lack of oxygen can lead to the activation of HIF-1. The HIF-1 transcriptional complex plays an important role in the regulation of oxygen homeostasis in mammalian cells (8-10). HIF-1 is a member of the basic helix-loop-helix-Per-Arnt-Sim superfamily, and is composed of an HIF- $1 \alpha$ and an HIF-1 $\beta$ subunit (11). HIF- $1 \alpha$ is regulated by oxygen levels and determines the level of HIF-1 activity, whereas HIF- $1 \beta$ is constitutively expressed, and its activity is not affected by hypoxia $(12,13)$. HIF-1 can affect many cellular processes, such as energy metabolism, tumor invasion, erythropoiesis, cell migration, angiogenesis and $\mathrm{pH}$ regulation, by controling the transcription of hundreds of HIF-mediated cells (14). The constitutive activation of HIF-1 inhibits mitochondrial biogenesis and cellular respiration by the repression of c-Myc activity in von Hippel-Lindau protein-deficient renal carcinoma cells (15). In 
addition, HIF-1 can affect tumor cell apoptosis (16). Zhang et al indicated that HIF-1 increased hypoxic cell survival under hypoxic conditions (17) and also suggested that HIF-1 activity may be involved in the protection of neuronal cells through the regulation of mitochondrial autophagy (17).

The mitochondria are replaced every 2-4 weeks in the rat liver, kidneys, heart and brain (18). Mitochondrial degradation has been implicated in the irreversible cell damage that may occur during cerebral ischemia and reperfusion (19). The destruction of the mitochondria may be the result of the process of autophagy, in which parts of the cytoplasm are sequestered in autophagosomes. A number of studies have suggested that autophagy, which is induced in the heart under hypoxic or ischemic conditions, plays either a protective or pathogenic role in heart disease (20-22). Moreover, autophagy may induce changes in mitochondrial mass. Mitochondrial mass can change either due to mitochondrial degradation or due to mitochondrial amplification, and it has been suggested that autophagy results in decreased mitochondrial mass through the accelerated mitochondrial degradation (23).

To the best of our knowledge, the present study provides the first direct evidence that the process of mitochondrial autophagy is dependent on the expression of HIF-1 under conditions of cerebral I/R. We demonstrate that mitochondrial autophagy protects neuronal cells, and the effects of HIF-1 on mitochondrial autophagy involve the inhibition of the mTOR signaling pathway.

\section{Materials and methods}

Animals. All procedures were carried out according to the protocols approved by the Ethics Committee for Animal Experimentation of the General Hospital of People's Liberation Army Chengdu Military Region (Chengdu, China). The Sprague-Dawley rats (210-240 g) that were used in this study were obtained from the Animal Center of the Institute of Field Surgery of the Third Military Medical University in China.

Primary cortical neuronal culture. Mouse cortical neurons were cultured according to a previously described method (24). Newborn (1-3 days old) Sprague-Dawley rats were anesthetized with halothane and sacrificed by cervical dislocation. The cortices obtained from the Sprague-Dawley rats were suspended in cold D-Hank's (HyClone, Salt Lake City, UT, USA) solution and dissected free of meninges and blood vessels. The cerebral tissues were digested in $0.125 \%$ trypsin for $30 \mathrm{~min}$ at $37^{\circ} \mathrm{C}$. The cell suspension was centrifuged at $3,000 \mathrm{x}$ g for $10 \mathrm{~min}$ at $4^{\circ} \mathrm{C}$, and the precipitate was re-suspended in DMEM/F12 medium (HyClone) supplemented with 20\% FBS (HyClone), $100 \mathrm{mg} / \mathrm{l}$ streptomycin and $100 \mathrm{kU} / 1$ benzylpenicillin. The cells were plated at $1 \times 10^{6} / \mathrm{ml}$ on 96 -well plates coated with $10 \mathrm{mg} / 1$ poly-Llysine. After $72 \mathrm{~h}, 5 \mu \mathrm{g} / \mathrm{ml}$ arabinosylcytosine were added to the cells to prevent the growth of non-neuronal cells. Subsequently, the culture medium was replaced with normal medium after $24 \mathrm{~h}$ and was refreshed every 2-3 days (25). The cultures typically contained $>95 \%$ neurons under these conditions.

In vitro simulation of I/R. Oxygen-glucose-deprivation (OGD) was induced according to a previously described method with minor modifications $(24,26)$. Briefly, the cells were rinsed twice with PBS and glucose-free Earl's solution [116.4 mmol/1 $\mathrm{NaC}_{1}$, $5.4 \mathrm{mmol} / 1 \mathrm{KCl}, 1.8 \mathrm{mmol} / 1 \mathrm{CaCl}_{2}, 0.8 \mathrm{mmol} / 1 \mathrm{MgSO}_{4}$, $2.6 \mathrm{mmol} / 1 \mathrm{NaH}_{2} \mathrm{PO}_{4}, 26.2 \mathrm{mmol} / 1 \mathrm{NaHCO}_{3}$ and $20.1 \mathrm{mmol} / \mathrm{l}$ HEPES (pH 7.4)] was added and the cells were cultured at $37^{\circ} \mathrm{C}$ in an incubator in an atmosphere of $5 \% \mathrm{CO}_{2}$ and $95 \% \mathrm{~N}_{2}$ (OGD) for $2 \mathrm{~h}$. OGD was terminated by the replacement of stored medium (Earl's solution, with the addition of $5.6 \mathrm{mmol} / \mathrm{l}$ glucose into the glucose-free Earl's solution) and by returning the cultures to an atmosphere of $5 \% \mathrm{CO}_{2}$ and $95 \% \mathrm{O}_{2}$ for another $12 \mathrm{~h}$.

Construction of clone and vector expressing HIF-1 $\alpha$. The Ad-CMV-HIF-1 $\alpha$ vector was constructed as described in a previous study (27). Briefly, total RNA was extracted from the cultured OGD-treated neurons using TRIzol reagent (Invitrogen Life Technologies, Carlsbad, CA, USA) according to the manufacturer's instructions. Approximately $5 \mu \mathrm{g}$ of total RNA was reverse transcribed into cDNA using a PrimeScript RT reagent kit (Takara Bio, Inc., Shiga, Japan). The cDNAs were used as templates for the amplification of HIF-1 $\alpha$ using 2 primers: forward, 5'-CGGTACCATGGAGGGCGCCGGCG GCGCG-3' and reverse, 5'-CGCGGCCGCTCAGTTAACTTG ATCCAAAGC-3'. The obtained sequences were fully sequenced (Shanghai Sangon Biotech Co., Ltd., Shanghai, China). The cloned HIF-1 $\alpha$ was inserted into a pENTR11 vector with KpnI and NotI restriction sites. Ad-CMV-HIF-1 $\alpha$ was generated using the ViraPower ${ }^{\mathrm{TM}}$ Adenoviral Expression System (Invitrogen Life Technologies) according to the operating protocols. Cells were seeded in 6 -well plates $\left(1.5 \times 10^{5}\right.$ cells/well $)$ and $100 \mu 1$ $\left(5 \times 10^{10} \mathrm{IU} / \mathrm{ml}\right)$ Ad-CMV-HIF-1 $\alpha$ were added and the cells were incubated at $37^{\circ} \mathrm{C}$ with $5 \% \mathrm{CO}_{2}$ for the indicated periods of time.

$q R T-P C R$. The transcription of HIF-1 $\alpha$ was detected using the RT-PCR method as previously described with minor modifications (28). The total RNA of primary neuronal cultures was isolated using TRIzol reagent according to the manufacturer's instructions (Biostar, Shanghai, China) and was then purified using the RNeasy mini kit (Qiagen, Palo Alto, CA, USA). Approximately $2 \mu \mathrm{g}$ of total RNA was reverse transcribed into first-strand cDNA using a QuantiTect ${ }^{\circledR}$ reverse transcription (RT) kit(Qiagen, Valencia,CA,USA) according to the manufacturer's instructions. qRT-PCR was performed to analyze the levels of mRNA transcripts using specific primers, using the QuantiTect ${ }^{\mathrm{TM}}$ SYBR $^{\circledR}$-Green PCR kit (Qiagen) and a Smart Cycler ${ }^{\circledR} 1.2 \mathrm{f}$ Detection System (Cepheid, Sunnyvale, CA, USA). The primer sequences used for qRT-PCR were as follows: HIF-1 $\alpha$ sense, 5'-CCAGCAGACTCAAATACAAGAACC-3' and antisense, 5'-TGTATGTGGGTAGGAGATGGAGAT-3'; $\beta$-actin sense, 5'-AAGCAGGAGTATGACGAGTCCG-3' and antisense, 5'-GCCTTCATACATCTCAAGTTGG-3'. These primers were all synthesized by Shanghai Sangon Biotech Co., Ltd. Cycling conditions were as follows: pre-incubation at $95^{\circ} \mathrm{C}, 15 \mathrm{~min}$; PCR: $95^{\circ} \mathrm{C}, 15 \mathrm{sec}$ and $56^{\circ} \mathrm{C}, 30 \mathrm{sec}, 40$ cycles; final elongation: $72^{\circ} \mathrm{C}, 10 \mathrm{~min}$. $\beta$-actin was used as an internal control and the expression levels of the relative genes were calculated using the $2^{-\Delta \Delta \mathrm{CT}}$ method, as previously described (29).

Western blot analysis. The total protein of the OGD-treated neurons was extracted using RIPA lysis buffer (Beyotime, Nantong, China) according to the manufacturer's instructions. A total of $40 \mu \mathrm{g}$ of protein per lane was subjected to $10 \%$ SDS-PAGE 
A

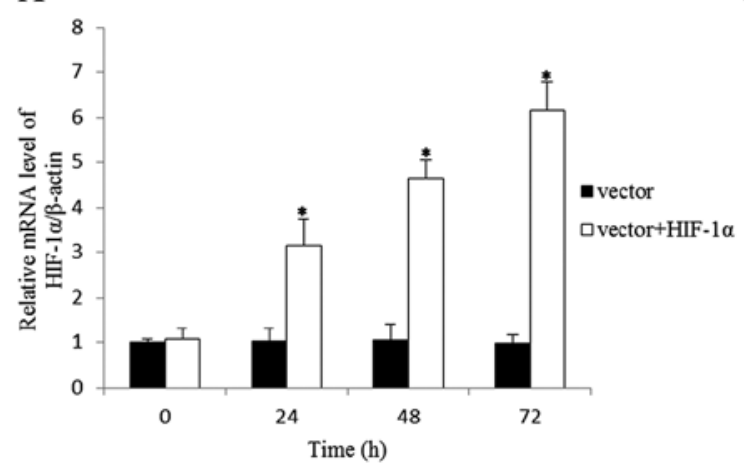

B

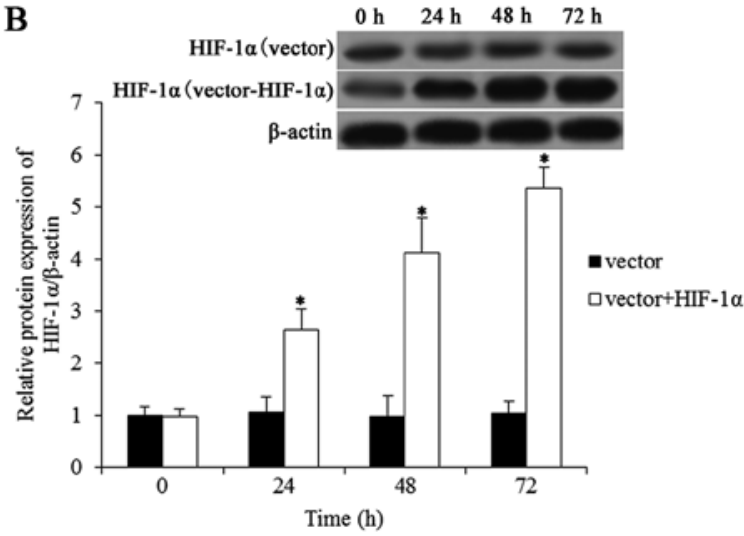

Figure 1. Hypoxia-inducible factor-1 $\alpha$ (HIF-1 $\alpha)$ mRNA and protein levels increased following transfection with Ad-CMV-HIF-1 $\alpha$. (A) The relative mRNA levels were determined by qRT-PCR and normalized to the housekeeping gene, $\beta$-actin. (B) Western blot analysis of HIF-1 $\alpha$ expression in the cells transfected with Ad-CMV-HIF-1 $\alpha$. Data are normalized to $\beta$-actin values and are expressed as fold changes of $\beta$-actin in the cells transfected with Ad-CMV-HIF-1 $\alpha$ relative to the control. The expression of HIF-1 $\alpha$ in the cells transfected with Ad-CMV-HIF-1 $\alpha$ groups was significantly higher compared with the cells transfected with the control vector. All experiments were repeated at least 3 times. Values are shown as the means $\pm \mathrm{SD}(\mathrm{n}=3)$. ${ }^{*} \mathrm{P}<0.05$ vs. control.

and electroblotted onto nitrocellulose membranes (Amersham Pharmacia Biotech, Freiburg, Germany). The immunodetection of HIF-1 $\alpha$, LC3, p70S6 kinase and $\beta$-actin was carried out using HIF-1- $\alpha$ antibody (Abcam, Cambridge, MA, USA), LC3 antibody (Abcam), phospho-p70S6 kinase antibody (Thr-389; Cell Signaling Technology, Inc., Danvers, MA, USA) and $\beta$-actin antibody (Abcam). Goat anti-rabbit IgG (Abcam) was used as the secondary antibody. The bound antibodies were visualized using LumiGLO $^{\circledR}$ reagent (Pierce Biotechnology, Inc., Rockford, IL, USA). All experiments were repeated 3 times.

Fluorescence-activated cell sorting (FACS) analysis. The analysis of mitochondrial mass was performed using a FACScan flow cytometer (BD Biosciences, San Jose, CA, USA) according to a previously described method with minor modifications. Briefly, the cells were stained with $1 \mu \mathrm{M}$ dichlorodihydrofluorescein diacetate, $10 \mathrm{nM}$ nonyl acridine orange (NAO), or $1 \mu \mathrm{M}$ ER-tracker ${ }^{\mathrm{TM}}$ Green dye, and then cultured in PBS solution supplemented with $5 \%$ fetal bovine serum at $37^{\circ} \mathrm{C}$ for $15 \mathrm{~min}$. The stained cells were analyzed using a FACScan flow cytometer. Apoptosis was measured by flow cytometry using an Annexin V-FITC/PI kit (BD Pharmingen, San Diego, CA, USA) according to the manufacturer's instructions.

MTT cell proliferation assay. Cortical neuronal proliferation was measured by MTT assay as previously described (30). Briefly, the cortical neurons were seeded into 96-well culture plates for 24, 48 and $72 \mathrm{~h}$, and then $5 \mathrm{mg} / \mathrm{ml}$ MTT $(20 \mu \mathrm{l})$ were added to the cells at $37^{\circ} \mathrm{C}$ for $4 \mathrm{~h}$. A total of $200 \mu \mathrm{l}$ of DMSO was added to solubilize the crystals. The OD value was measured at a wavelength of $490 \mathrm{~nm}$ using a spectrophotometer (Multiskan MK3; Thermo Scientific, Waltham, MA, USA). Data are expressed as the means \pm standard error of the mean (SEM). Differences between the treatment groups were analyzed using the Student's t-test and a value of $\mathrm{P} \leq 0.05$ was considered to indicate a statistically significant difference.

Measurement of ATP levels. ATP levels in the neurons were determined using recombinant firefly luciferase and its substrate, D-luciferin (Invitrogen Life Technologies) according to the manufacturer's instructions and as previously described (31). ATP levels were quantitatively detected, and luminescence was measured using a luminometer (emission maximum at $560 \mathrm{~nm}$ ).

Transfection with small interfering RNA (siRNA). The neuronal cells expressing the HIF-1 $\alpha$ protein were transfected with HIF-1 $\alpha$ siRNA (siHIF-1 $\alpha$ ) or scrambled siRNA (siMock) using Lipofectamine 2000 (Invitrogen Life Technologies) according to the manufacturer's instructions. For the siRNA experiments, the HIF-1 $\alpha$ overexpressing cells and the wild-type control cells were seeded on 60-mm plates. The cells were transfected and, after $20 \mathrm{~h}$, were divided into 24 -well plates. The cells were analyzed 3 days after transfection. The siRNA oligo sequences for HIF-1 $\alpha$ were as previously described $(32,33)$. The siHIF-1 $\alpha$ target sequences were: sense, 5'-CUGAUGA CCAGCAACUUGAdTdT-3' and antisense, 5'-UCAAGUUGC UGGUCAUCAGdTdT-3'.

Statistical analysis. All values are expressed as the means \pm SEM. Statistical analysis was performed using the Student's t-test. A value of $\mathrm{P}<0.05$ was considered to indicate a statistically significant difference. All experiments were repeated at least 3 times.

\section{Results}

HIF-1a levels were increased in neurons transfected with $A d-C M V$ - $H I F-1 \alpha$.In order to induce the overexpression of HIF-1 $\alpha$ in primary cortical neurons, we created an adenoviral vector that encodes HIF-1 $\alpha$ under normoxic conditions. The expression of HIF-1 $\alpha$ in the cortical neurons was determined by qRT-PCR and western blot analysis. The qRT-PCR results (Fig. 1A) showed that the HIF-1 $\alpha$ mRNA levels in the neurons transfected with Ad-CMV-HIF-1 $\alpha$ were markedly higher than those of the control cells $24 \mathrm{~h}$ later. The protein expression was also determined and the results revealed that the HIF- $1 \alpha$ protein levels were upregulated in the neurons transfected with Ad-CMV-HIF-1 $\alpha$ (Fig. 1B). 

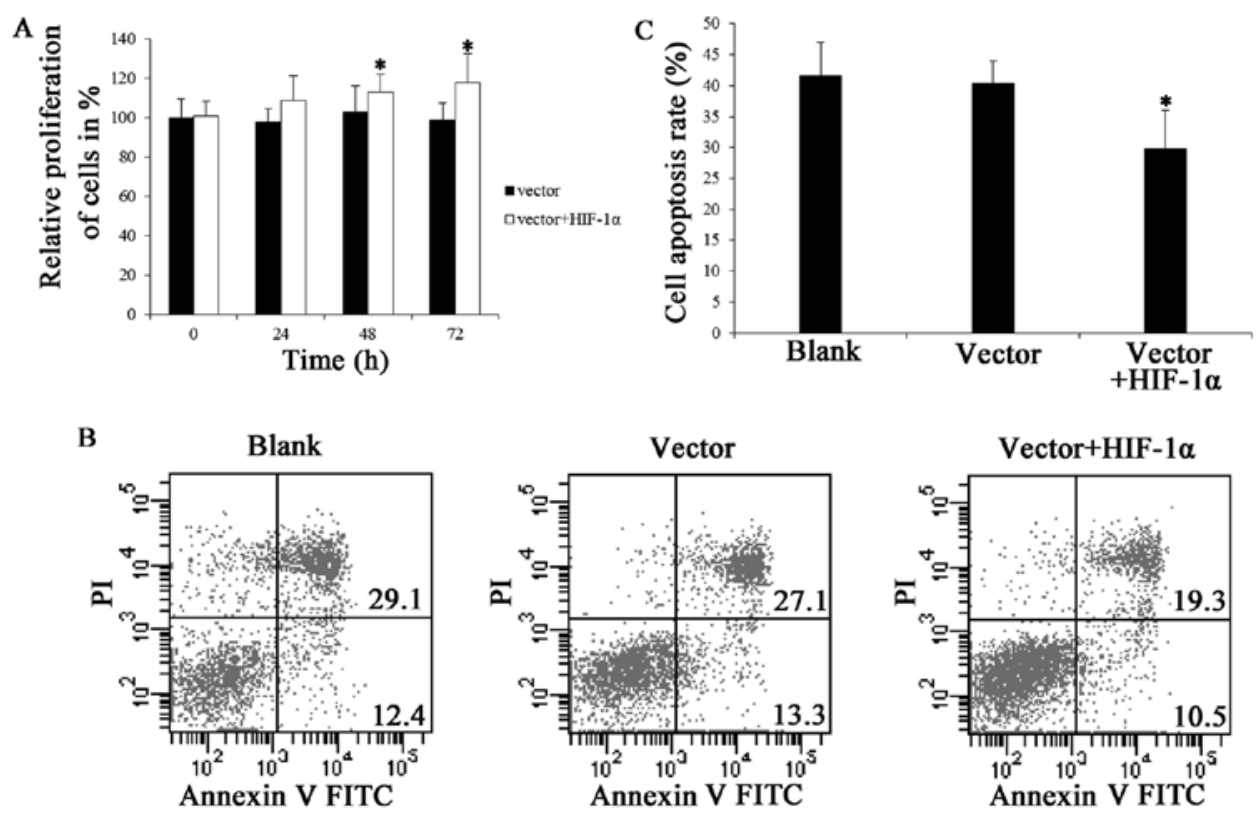

Figure 2. Hypoxia-inducible factor-1 $\alpha$ (HIF-1 $\alpha$ ) overexpression increased cell proliferation and decreased cell apoptosis. (A) HIF-1 $\alpha$ overexpression increased cell proliferation in a time-dependent manner. Cell proliferation was determined by MTT assay. Cells were transfected with Ad-CMV-HIF-1 $\alpha$ or the control vector for 24,48 or $72 \mathrm{~h}$. (B) Effect of HIF-1 $\alpha$ overexpression on cell apoptosis. Flow cytometric analysis of HIF-1 $\alpha$-overexpressing cells stained with Annexin V and PI. (C) Cell apoptotic rate. Flow cytometric analysis of HIF-1 $\alpha$-overexpressing cells stained with Annexin V and PI. Cells were transfected with Ad-CMV-HIF-1 $\alpha$ or the control vector for $72 \mathrm{~h}$. All experiments were repeated at least 3 times. The 'blank' group indicated untreated cells. The 'vector' group indicated cells infected with the empty plasmid packed in adenovirus. Values are shown as the means $\pm \mathrm{SD}(\mathrm{n}=3)$. ${ }^{*} \mathrm{P}<0.05$ vs. control.

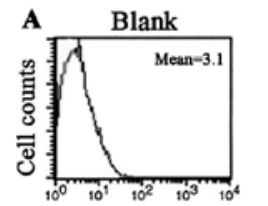

Fluorescence (AU)

$$
\mathbf{B}
$$
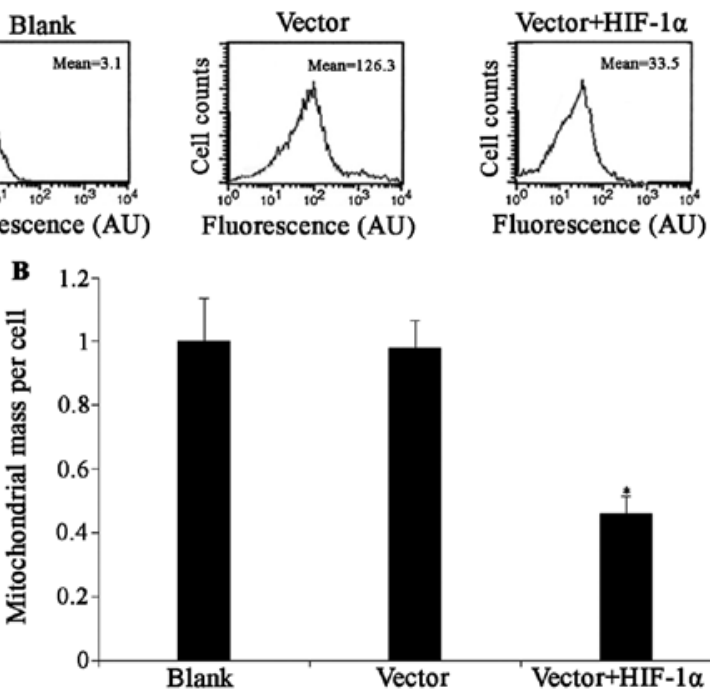

Figure 3. Evaluation of mitochondrial mass in the model of oxygen-glucosedeprivation (OGD)/RP using cultured neonatal rat cortical neurons by fluorescence-activated cell sorting (FACS) analysis. Cells were transfected with hypoxia-inducible factor-1 $\alpha$ (HIF-1 $\alpha$ )-expressing vector (Ad-CMVHIF-1 $\alpha$ ) or with the control vector. (A) FACS analysis. Mitochondria were labeled with MitoTracker Green (MTG), and signals were measured using the FACS analyzer. Cells were analyzed at $72 \mathrm{~h}$ after transfection. (B) Summary of mitochondrial mass by FACS analysis. Cells were analyzed at $72 \mathrm{~h}$ after transfection. Mean values were converted to arbitrary units (AU) to make the results from different experiments directly comparable with each other. All experiments were repeated at least 3 times. Values are shown as the means $\pm \mathrm{SD} n=3) .{ }^{*} \mathrm{P}<0.05$ vs. control.

Effect of HIF-1 $\alpha$ overexpression on neuronal cell proliferation and apoptosis. The effect of HIF-1 $\alpha$ overexpression on neuronal cell proliferation was determined by MTT assay.
The results indicated that there was an increase in the proliferation of cortical neurons which overexpressed HIF-1 $\alpha$ and were subjected to OGD, and the difference was significant following transfection with HIF-1 $\alpha$ for $48 \mathrm{~h}$; however, there was no significant difference at $24 \mathrm{~h}$ (Fig. 2A). We found that HIF-1 $\alpha$ overexpression led to an almost $18 \%$ increase in the number of cells. This indicated that HIF-1 $\alpha$ exerts positive effects by increasing the number of cells.

It has recently been demonstrated that HIF-1 exerts an anti-apoptotic effect on p53-mediated apoptosis through a secreted neuronal tyrosinase (34). Moreover, HIF-1 can induce erythropoietin production under hypoxic and hypoglycemic conditions, and erythropoietin can prevent neuronal apoptosis following cerebral ischemia and metabolic stress $(35,36)$. Thus, we examined whether the cell increase in our experiments was mediated by apoptosis. Apoptosis was first examined by flow cytometric analysis of the cells stained with Annexin V and PI. The results revealed that HIF-1 $\alpha$ overexpression prevented cell apoptosis, and that the apoptotic rate decreased to $29.8 \%$ when the cells were transfected with Ad-CMV-HIF-1 $\alpha$ for $72 \mathrm{~h}$ (Fig. 2B and C).

Effect of HIF-1 $\alpha$ overexpression on mitochondrial mass in cortical neurons. The above-mentioned results suggested that HIF-1 $\alpha$ overexpression protected neurons against apoptosis; however, the mechanisms involved remain unclear. The constitutive activation of HIF-1 inhibits mitochondrial biogenesis by repressing c-Myc activity in von Hippel-Lindau protein-deficient renal carcinoma cells (15). In addition, Zhang et al also suggested that HIF-1 activity may be involved in protecting neuron cells through the regulation of mitochondrial autophagy (17). We first evaluated mitochondrial mass using FACS analysis. Living cells 
A

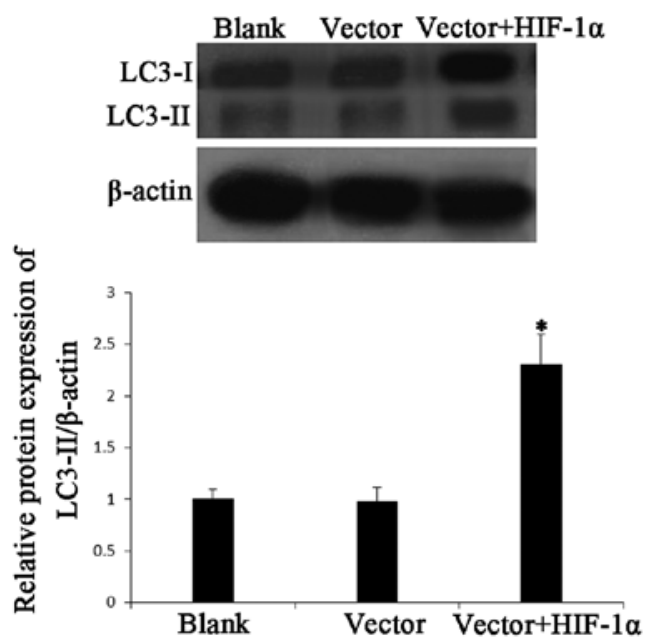

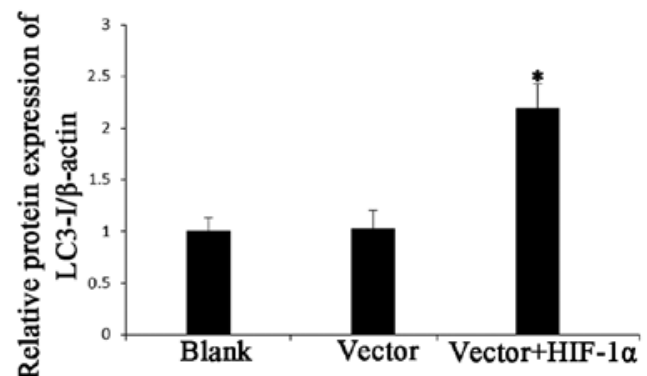

B

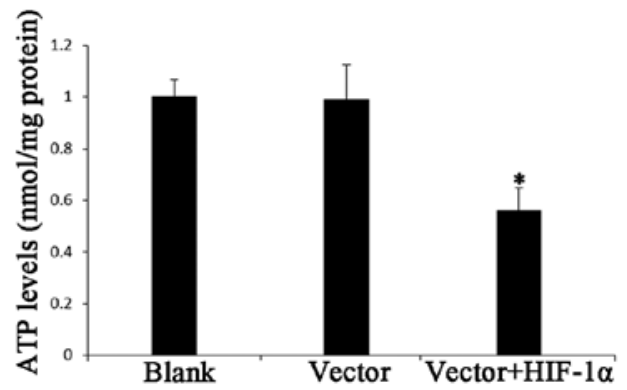

Figure 4. Hypoxia-inducible factor-1 $\alpha$ (HIF-1 $\alpha$ ) overexpression induced mitochondrial autophagy. (A) Levels of LC3 (I and II) were increased after the induction of HIF-1 $\alpha$ overexpression. Cells were transfected with HIF-1 $\alpha$ vector carrying the HIF-1 $\alpha$ fragment or with the control vector. Cells were analyzed at $72 \mathrm{~h}$ after transfection. Data were normalized to $\beta$-actin values and are expressed as fold changes of $\beta$-actin in HIF-1 $\alpha$ overexpression relative to the control. (B) ATP levels were decreased after the induction of HIF-1 $\alpha$ overexpression. All experiments were repeated at least 3 times. Values are shown as the means \pm SD ( $n=3$ ). ${ }^{*} \mathrm{P}<0.05$ vs. control.

were stained with MitoTracker Green (MTG), a fluorescent vital stain specific for mitochondria (23). The cells were transfected using the HIF-1 $\alpha$ adenoviral construct and analyzed at several time points following transfection. We found that the overexpression of HIF-1 $\alpha$ for $24 \mathrm{~h}$ or longer caused a significant decrease in mitochondrial mass compared with the control group (Fig. 3).

Effect of HIF-1 $\alpha$ overexpression on mitochondrial autophagy. Mitochondrial mass can change due to mitochondrial degradation, and autophagy results in a decrease in mitochondrial mass through accelerated mitochondrial degradation (23). We subsequently detected the effect of HIF-1 $\alpha$ overexpression on mitochondrial autophagy. Certain studies have demonstrated that LC3 (ATG8 in yeast) plays a central role in the autophagy pathway and promotes the formation of autophagosomes $(37,38)$. LC3 is known to exist in a soluble form, termed LC3-I and a lipidated form termed, LC3-II that is associated with autophagosomal membranes (39). LC3 levels in the cortical neurons following the induction of HIF-1 $\alpha$ overexpression were detected by western blot analysis. The levels of LC3-I and LC3-II were significantly increased in the cortical neurons following the induction of the overexpression of HIF-1 $\alpha$ for $24 \mathrm{~h}$ (data not shown), and maximally at $72 \mathrm{~h}$ (Fig. 4A). Certain studies have shown that mitochondrial autophagy is associated with a change in the ATP levels $(17,23)$. Thus, we also determined whether HIF-1 $\alpha$ regulates ATP levels. The overexpression of HIF-1 $\alpha$ for 24, 48 (data not shown) and $72 \mathrm{~h}$ led to significantly decreased ATP levels (Fig. 4B). These results are consistent with the above-mentioned findings that HIF-1 $\alpha$ leads to mitochondrial degradation.

Specific inhibition of HIF-1 $\alpha$ mRNA and protein expression by HIF-1 $\alpha$-specific siRNA. The above-mentioned experiments were designed to examine the effect of the overexpression of HIF-1 $\alpha$ in cortical neurons. We subsequently employed a complementary approach using RNA interference technology and examined the effects of suppressing the levels of HIF-1 $\alpha$ in the HIF-1 $\alpha$-overexpressing cells. The cells were transfected with siHIF-1 $\alpha$ or control siRNA (siMock) and were analyzed after 2 days of growth. Compared with the siMock-transfected cells, the levels of HIF- $1 \alpha$ in the cells transfected with siHIF-1 $\alpha$ were significantly decreased (Fig. 5A and B). We then determined the effect of HIF-1 $\alpha$ silencing on cell proliferation and mitochondrial autophagy. As shown in Fig. 5C, there was a decrease in the growth of the siHIF-1 $\alpha$-transfected cells compared with the siMock-transfected cells. Moreover, the LC3 protein levels were also decreased in the siHIF-1 $\alpha$-transfected cells (Fig. 5D). These results indicated that the increase in cell growth and the activity of mitochondrial autophagy in cortical neurons was associated with the overexpression of HIF-1 $\alpha$.

Overexpression of $H I F-1 \alpha$ is directly related to the inhibition of $m T O R$. There is evidence that the induction of autophagy is mediated by the inhibition of the mTOR pathway (40). Since HIF-1 $\alpha$ induces autophagy, we examined whether it also inhibits mTOR signaling. The expression of p70S6 kinase was detected by western blot analysis. p70S6 kinase expression was significantly increased in the siHIF-1 $\alpha$-transfected cells compared with siMock group (Fig. 6). These results demonstrated that HIF-1 $\alpha$ overexpression inhibited the mTOR pathway.

\section{Discussion}

The main findings of the present study were the following: i) the overexpression of HIF-1 $\alpha$ improves cell survival in the OGD/RP model using cultured neonatal rat cortical neurons; ii) the overexpression of HIF-1 $\alpha$ significantly induces mitochondrial autophagy and degradation in the OGD/RP model 

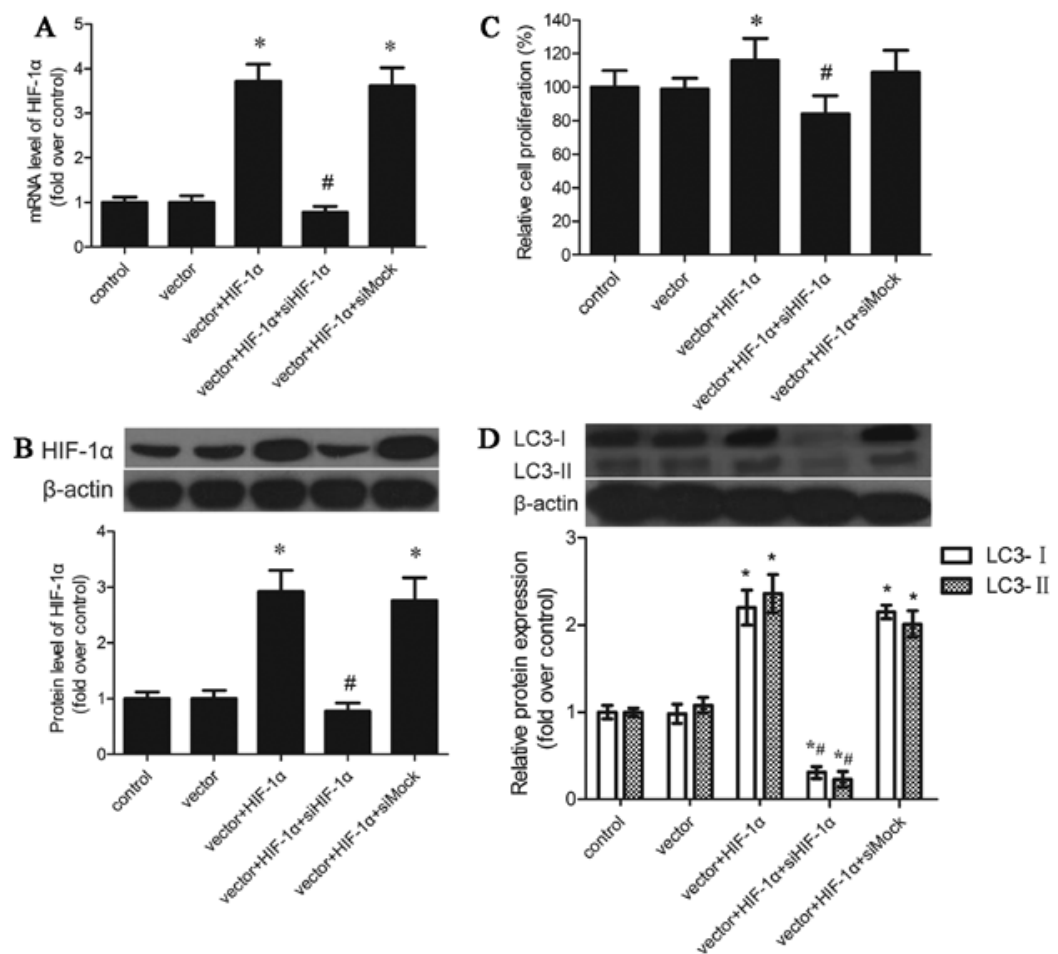

Figure 5. Effect of HIF-1 $\alpha$ siRNA (siHIF-1 $\alpha$ )-transfection on cell proliferation and mitochondrial autophagy. (A) The proliferation of cells transfected with siHIF-1 $\alpha$ or scrambled siRNA (siMock) cells after the induction of hypoxia-inducible factor-1 $\alpha$ (HIF-1 $\alpha$ ) overexpression. (B) Western blot analysis of LC3-I and LC3-II expression. LC3-I and LC3-II expression levels were normalized to $\beta$-actin. The 'control group' indicates wild-type untreated cells. The 'vector' group indicates cells infected with the empty plasmid packed in adenovirus. All experiments were repeated at least 3 times. Values are shown as the means $\pm \mathrm{SD}(\mathrm{n}=3) .{ }^{*} \mathrm{P}<0.05$ vs. control; ${ }^{*} \mathrm{P}<0.05$ vs. vector $+\mathrm{HIF}-1 \alpha$.

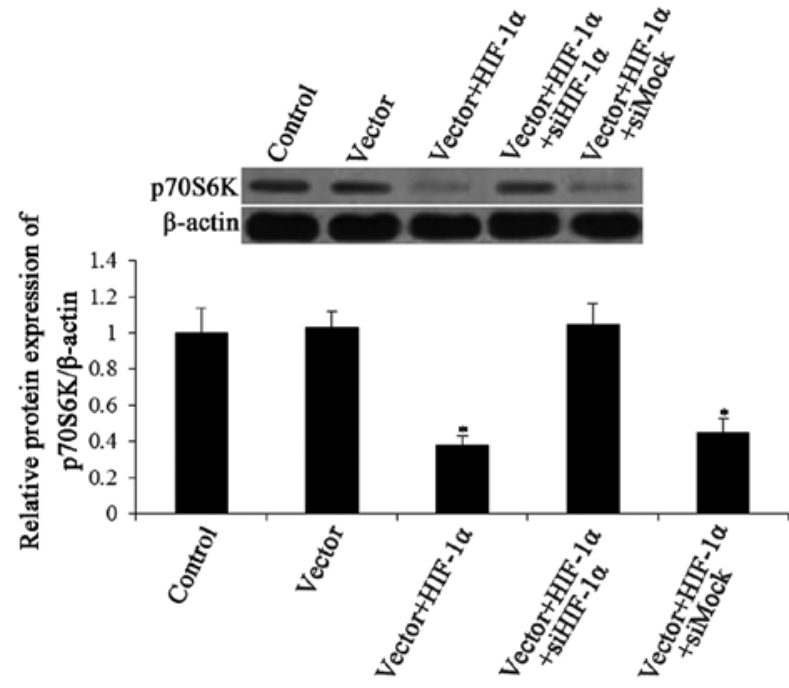

Figure 6. Hypoxia-inducible factor-1 $\alpha$ (HIF-1 $\alpha$ ) overexpression inhibits mTOR activation. The expression of p70S6 kinase (p70S6K) was detected by western blot analysis. The expression of p70S6K was markedly reduced in the HIF-1 $\alpha$-overexpressing cells compared with the control cells, whereas the p70S6K level was significantly increased after HIF-1 $\alpha$ silencing. Data were normalized to $\beta$-actin values and expressed as fold changes of $\beta$-actin. All experiments were repeated at least 3 times. Values are shown as the means $\pm \mathrm{SD}(\mathrm{n}=3) .{ }^{*} \mathrm{P}<0.05$ vs. control.

using cultured neonatal rat cortical neurons; iii) the inhibition of HIF-1 $\alpha$ markedly suppresses mitochondrial autophagy in rat cortical neurons; and iv) the overexpression of HIF-1 $\alpha$ induced mitochondrial autophagy and this was accompanied by the inhibition of the mTOR pathway. To the best of our knowledge, these data demonstrate for the first time that the neuroprotective effects of the overexpression of HIF-1 $\alpha$ in a model of OGD/RP using cultured neonatal rat cortical neurons involve an increase in mitochondrial autophagy. Thus, the results provide insight into the role of HIF-1 $\alpha$ in cerebral I/R injury.

In the present study, we analyzed the effects of HIF-1 $\alpha$ overexpression. HIF-1 is a major regulator of oxygen homeostasis (10). It is composed of 2 subunits, an $\alpha$ and a $\beta$ subunit, but the regulation of HIF- 1 activity mostly depends on the $\alpha$ subunit. The upregulation of HIF-1 and the increased HIF-1 binding activity have been shown to protect astrocytes from ischemic injury during $6 \mathrm{~h}$ of hyperthermia $\left(38\right.$ or $\left.40^{\circ} \mathrm{C}\right)(41)$. Moreover, the upregulation of HIF- $1 \alpha$ and the increased binding activity of HIF-1 are associated with the neuroprotective effects induced by hypoxia/ischemia pre-conditioning (42). The present study demonstrates that the overexpression of HIF-1 $\alpha$ improves cell survival in a model of OGD/RP using cultured neonatal rat cortical neurons. We found that a high level of HIF-1 $\alpha$ is associated with an increase in cell proliferation and a decrease in cell apoptosis. These data suggest that the upregulation of the level of HIF-1 $\alpha$ protects cortical neurons from ischemia-reperfusion injury.

The present study also demonstrates that the overexpression of HIF-1 $\alpha$ significantly induces changes in mitochondrial mass in cortical neurons. Changes in mitochondrial mass may be due to mitochondrial degradation, and autophagy results in a decrease in the mitochondrial mass through accelerated mitochondrial degradation (23). Thus, we also examined the 
effect of HIF-1 $\alpha$ overexpression on mitochondrial autophagy. LC3 (ATG8 in yeast) is a well-accepted marker of increased autophagy, plays a central role in the autophagy pathway and can promote the formation of autophagosomes $(37,38)$. Our study demonstrated that HIF-1 $\alpha$ overexpression is followed by increased LC3 protein levels, particularly LC3-II. Our data indicated that HIF-1 $\alpha$ overexpression induced mitochondrial autophagy. The present study also demonstrated that the inhibition of HIF-1 $\alpha$ expression markedly suppressed cortical neuron survival and mitochondrial autophagy. A previous study demonstrated that mitochondrial autophagy is dependent on the level of HIF-1 (17). We found that siHIF-1 $\alpha$ suppressed cell survival and increased the apoptotic rate. Moreover, we demonstrated that HIF-1 $\alpha$ silencing also inhibited mitochondrial autophagy. In addition, we found that HIF-1 $\alpha$ induced mitochondrial autophagy through the inhibition of the mTOR pathway.

In conclusion, the present results indicate that HIF-1 $\alpha$ overexpression increases cell survival in a model of OGD/RP using cultured neonatal rat cortical neurons. Moreover, HIF-1 $\alpha$ overexpression increased mitochondrial autophagy. The inhibition of the expression of HIF-1 $\alpha$ suppressed cell survival and decreased mitochondrial autophagy. It is, therefore, conceivable that HIF-1 $\alpha$ targeting may have detrimental effects on cortical neurons subjected to OGD/RP. More importantly, our findings raise the possibility that HIF- $1 \alpha$ may serve as a novel regulator of cerebral I/R injury.

\section{Acknowledgements}

This study was supported by the Project of General Hospital of the People's Liberation Army Chengdu Military Region (2013YG-A010).

\section{References}

1. Rosamond W, Flegal K, Furie K, et al: Heart disease and stroke statistics--2008 update: a report from the American Heart Association Statistics Committee and Stroke Statistics Subcommittee. Circulation 117: e25-e146, 2008.

2. Wass CT and Lanier WL: Glucose modulation of ischemic brain injury: review and clinical recommendations. In: Mayo Clin Proc, 801-812, 1996.

3. Hackett ML, Duncan JR, Anderson CS, Broad JB and Bonita R: Health-related quality of life among long-term survivors of stroke: results from the Auckland Stroke Study, 1991-1992. Stroke 31: 440-447, 2000.

4. Nitatori T, Sato N, Waguri S, et al: Delayed neuronal death in the CA1 pyramidal cell layer of the gerbil hippocampus following transient ischemia is apoptosis. J Neurosci 15: 1001-1011, 1995.

5. Halterman MW and Federoff HJ: HIF- $1 \alpha$ and p53 promote hypoxia-induced delayed neuronal death in models of CNS ischemia. Exp Neurol 159: 65-72, 1999.

6. Halterman MW, Miller CC and Federoff HJ: Hypoxia-inducible factor-1alpha mediates hypoxia-induced delayed neuronal death that involves p53. J Neurosci 19: 6818-6824, 1999.

7. Chen CJ, Cheng FC, Liao SL, Chen WY, Lin NN and Kuo JS: Effects of naloxone on lactate, pyruvate metabolism and antioxidant enzyme activity in rat cerebral ischemia/reperfusion. Neurosci Lett 287: 113-116, 2000.

8. Semenza GL: HIF-1: mediator of physiological and pathophysiological responses to hypoxia. J Appl Physiol 88: 1474-1480, 2000

9. Melillo G: HIF-1: a target for cancer, ischemia and inflammation--too good to be true? Cell Cycle 3: 149-150, 2004

10. Brahimi-Horn MC and Pouysségur J: Oxygen, a source of life and stress. FEBS Lett 581: 3582-3591, 2007.

11. Wang GL, Jiang B-H and Semenza GL: Effect of protein kinase and phosphatase inhibitors on expression of hypoxia inducible factor 1. Biochem Biophys Res Commun 216: 669-675, 1995.
12. Huang LE, Gu J, Schau M and Bunn HF: Regulation of hypoxia-inducible factor lalpha is mediated by an $\mathrm{O}_{2}$-dependent degradation domain via the ubiquitin-proteasome pathway. Proc Natl Acad Sci USA 95: 7987, 1998.

13. Wang GL,Jiang B-H,RueEA and Semenza GL: Hypoxia-inducible factor 1 is a basic-helix-loop-helix-PAS heterodimer regulated by cellular $\mathrm{O}_{2}$ tension. Proc Natl Acad Sci USA 92: 5510-5514, 1995.

14. Mazure NM and Pouysségur J: Hypoxia-induced autophagy: cell death or cell survival? Current opinion in cell biology 22: 177-180, 2010.

15. Zhang H, Gao P, Fukuda R, et al: HIF-1 inhibits mitochondrial biogenesis and cellular respiration in VHL-deficient renal cell carcinoma by repression of C-MYC activity. Cancer Cell 11: 407-420, 2007.

16. Sowter HM, Ratcliffe PJ, Watson P, Greenberg AH and Harris AL: HIF-1-dependent regulation of hypoxic induction of the cell death factors BNIP3 and NIX in human tumors. Cancer Res 61: 6669-6673, 2001.

17. Zhang H, Bosch-Marce M, Shimoda LA, et al: Mitochondrial autophagy is an HIF-1-dependent adaptive metabolic response to hypoxia. J Biological Chem 283: 10892-10903, 2008.

18. Menzies RA and Gold PH: The turnover of mitochondria in a variety of tissues of young adult and aged rats. J Biol Chem 246: 2425-2429, 1971.

19. Rosenthal RE, Hamud F, Fiskum G, Varghese PJ and Sharpe S: Cerebral ischemia and reperfusion: prevention of brain mitochondrial injury by lidoflazine. J Cereb Blood Flow Metab 7: 752-758, 1987.

20. Mei Y, Thompson MD, Cohen RA and Tong X: Autophagy and oxidative stress in cardiovascular diseases. Biochim Biophys Acta May 13, 2014 (Epub ahead of print).

21. Ma S, Wang Y, Chen Y and Cao F: The role of the autophagy in myocardial ischemia/reperfusion injury. Biochim Biophys Acta May 21, 2014 (Epub ahead of print).

22. Ghavami S, Gupta S, Ambrose EC, Hnatowich M, Freed DH and Dixon IM: Autophagy and Heart Disease: Implications for Cardiac Ischemia-Reperfusion Damage. Curr Mol Med: June 2, 2014 (Epub ahead of print).

23. Ermak G, Sojitra S, Yin F, Cadenas E, Cuervo AM and Davies KJ: Chronic expression of RCAN1-1L protein induces mitochondrial autophagy and metabolic shift from oxidative phosphorylation to glycolysis in neuronal cells. J Biol Chem 287: 14088-14098, 2012 .

24. Gong QH, Wang Q, Shi JS, Huang X-n, Liu Q and Ma H: Inhibition of caspases and intracellular free $\mathrm{Ca} 2+$ concentrations are involved in resveratrol protection against apoptosis in rat primary neuron cultures. Acta Pharmacol Sin 28: 1724-1730, 2007.

25. Murphy TH and Baraban JM: Glutamate toxicity in immature cortical neurons precedes development of glutamate receptor currents. Brain Res Dev Brain Res 57: 146-150, 1990.

26. Choi DW, Maulucci-Gedde M and Kriegstein AR: Glutamate neurotoxicity in cortical cell culture. J Neurosci 7: 357-368, 1987.

27. Wei L, Lu J, Feng L, Li S, Shan J and Li Y: Construction of recombinant adenovirus vector containing a modified gene that codes for human hypoxia-inducible factor- $1 \alpha$ without oxygen-dependent degradation domain. Plasmid 63: 20-26, 2010.

28. Bryant CS, Munkarah AR, Kumar S, et al: Reduction of hypoxia-induced angiogenesis in ovarian cancer cells by inhibition of HIF-1 alpha gene expression. Arch Gynecol Obstet 282: 677-683, 2010.

29. Livak K and Schmittgen T: Analysis of relative gene expression data using real-time quantitative PCR and the 2(-Delta Delta C(T)) Method. Methods 25: 402-408, 2001.

30. Song YX, Yue ZY, Wang ZN, et al: MicroRNA-148b is frequently down-regulated in gastric cancer and acts as a tumor suppressor by inhibiting cell proliferation. Mol Cancer 10: 1, 2011.

31. Zadran S, Sanchez D, Zadran H, Amighi A, Otiniano E and Wong K: Enhanced-acceptor fluorescence-based single cell ATP biosensor monitors ATP in heterogeneous cancer populations in real time. Biotechnol Lett 35: 175-180, 2013.

32. Sowter HM, Raval R, Moore J, Ratcliffe PJ and Harris AL: Predominant role of hypoxia-inducible transcription factor (Hif) $-1 \alpha$ versus Hif- $2 \alpha$ in regulation of the transcriptional response to hypoxia. Cancer Res 63: 6130-6134, 2003.

33. Esteban MA, Tran MG, Harten SK, et al: Regulation of E-cadherin expression by VHL and hypoxia-inducible factor. Cancer Res 66: 3567-3575, 2006.

34. Sendoel A, Kohler I, Fellmann C, Lowe SW and Hengartner MO: HIF-1 antagonizes p53-mediated apoptosis through a secreted neuronal tyrosinase. Nature 465: 577-583, 2010. 
35. Sirén AL, Fratelli M, Brines M, et al: Erythropoietin prevents neuronal apoptosis after cerebral ischemia and metabolic stress. Proc Natl Acad Sci USA 98: 4044-4049, 2001.

36. Chandel N, Maltepe E, Goldwasser E, Mathieu C, Simon M and Schumacker P: Mitochondrial reactive oxygen species trigger hypoxia-induced transcription. Proc Natl Acad Sci USA 95: $11715-11720,1998$.

37. Levine B, Mizushima N and Virgin HW: Autophagy in immunity and inflammation. Nature 469: 323-335, 2011.

38. Geng J and Klionsky DJ: The Atg8 and Atg12 ubiquitin-like conjugation systems in macroautophagy. EMBO Rep 9: 859-864, 2008.

39. Kraft LJ and Kenworthy AK: Imaging protein complex formation in the autophagy pathway: analysis of the interaction of LC3 and Atg4BC74A in live cells using Förster resonance energy transfer and fluorescence recovery after photobleaching. J Biomed Opt 17: 0110081-01100812, 2012.
40. Mijaljica D, Prescott M and Devenish RJ: Different fates of mitochondria: alternative ways for degradation? Autophagy 3 : 4-9, 2007.

41. DuF,ZhuL, QianZM,WuXM, Yung WH and Ke Y:Hyperthermic preconditioning protects astrocytes from ischemia/reperfusion injury by up-regulation of HIF-1 alpha expression and binding activity. Biochim Biophys Acta 1802: 1048-1053, 2010.

42. Du F, Wu XM, Gong Q, He X and Ke Y: Hyperthermia conditioned astrocyte-cultured medium protects neurons from ischemic injury by the up-regulation of HIF-1 alpha and the increased antiapoptotic ability. Eur J Pharmacol 666: 19-25, 2011. 